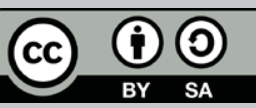

Jiménez, I. (2021). Elementos que identifican los métodos comparados. Collectivus. Revista de Ciencias Sociales, 8 (2), 167-192.

https://doi.org/10.15648/Collectivus.vol8num2.2021.3134

doi

\title{
ShcolLECTIVLS
}

REVISTA DE CIENCIAS SOCIALES

\section{Elementos que identifican los Métodos Comparados}

Features identifying the Methods Compared

IVONNE JIMENEZ JIMENEZ*

iD HTTPS://ORCID.ORG/0000-0003-4070-5294

* Doctora en Educación. Universidad Cuauhtémoc México, docente secretaria de Educación de Cundinamarca. ivjiji@hotmail.com. 


\section{RES U M E N}

La comparación como método de investigación desde las Ciencias Sociales posee unos elementos que lo identifican, entre ellos: indagar sobre política, diagnosticar problemas sociales, describir, explicar e interpretar la realidad. A razón de lo anterior, para ilustrar el método comparado, este artículo de reflexión teórica tiene por objetivo analizar los principales elementos que constituyen el método comparado, para ello, desarrolla una triada teórico-metodológica que puntualiza sobre: los elementos de la comparación y el método comparado, la metodología de la comparación y unas reflexiones finales para investigaciones basadas en el método comparado, permitiendo de este modo tener entre las conclusiones como bien lo señala Sartori (1994) que el método comparado conlleva a asimilar y diferenciar en lo limites ya que no todo es comparable.

Palabras clave: comparar, metodología de la comparación, técnicas para la comparación.

\section{A B S T R A C T}

Comparison as a research method from the Social Sciences has some elements that identify it, among them: to inquire about politics, diagnose social problems, describe, explain, and interpret reality. Due to the above, in order to illustrate the comparative method, this article of theoretical reflection aims to analyze the main elements that constitute the comparative method, for this purpose, it develops a theoretical-methodological triad that points out: the elements of comparison and the comparative method, the methodology of comparison and some final reflections for research based on the comparative method, thus allowing to have among the conclusions as Sartori (1994) points out that the comparative method leads to assimilate and differentiate in the limits since not everything is comparable.

Keywords: compare, comparison methodology, techniques for comparison 


\section{Éléments qui identifient les méthodes comparatives}

\section{RÉ S U M É}

La comparaison en tant que méthode de recherche dans les sciences sociales se caractérise par un certain nombre d'éléments, notamment: l'enquête sur la politique, le diagnostic des problèmes sociaux, la description, l'explication et l'interprétation de la réalité. Pour cette raison, afin d'illustrer la méthode comparative, cet article de réflexion théorique vise à analyser les principaux éléments qui constituent la méthode comparative, en développant une triade théorique-méthodologique qui met en évidence: les éléments de la comparaison et la méthode comparative, la méthodologie de la comparaison et quelques réflexions finales pour la recherche basée sur la méthode comparative, permettant ainsi d'avoir parmi les conclusions comme Sartori (1994) souligne que la méthode comparative conduit à assimiler et différencier dans les limites, puisque tout n'est pas comparable.

Mots-clés: comparaison, méthodologie de comparaison, techniques de comparaison.

\section{Elementos que identificam os Métodos Comparativos}

\section{RES U M O}

A comparação como método de investigação nas ciências sociais tem uma série de elementos que a identificam, entre os quais: inquirir sobre política, diagnosticar problemas sociais, descrever, explicar e interpretar a realidade. Por esta razão, para ilustrar o método comparativo, este artigo de reflexão teórica visa analisar os principais elementos que constituem o método comparativo, desenvolvendo uma tríade teórico-metodológica que aponta: os elementos de comparação e o método comparativo, a metodologia de comparação e algumas reflexões finais para a investigação baseada no método comparativo, permitindo assim ter entre as conclusões como Sartori (1994) assinala que o método comparativo leva a assimilar e diferenciar nos limites, uma vez que nem tudo é comparável.

Palavras chave: comparação, metodologia de comparação, técnicas de comparação. 



\section{Introducción}

En la actualidad se puede evidenciar desde diversas disciplinas la aplicación del EC ${ }^{1}$ como metodología de investigación, lo anterior, por razones como las que plantea (Flyvbjerg, 2004) quien dice que el EC es importante ya que proporciona una visión matizada de la realidad, de ahí: la medicina ha podido explicar diversas patologías en enfermedades, la Sociología con Weber (1904) analizar las relaciones del protestantismo con el surgimiento del capitalismo; con Merton (1970) el problema de las relaciones entre el puritanismo y el desarrollo de la ciencia en Inglaterra, con Durkheim (1912) analizar las formas elementales de la vida religiosa. También, vale la pena señalar que, en educación el EC ha sido aplicado por Stake, (1999) quien dirigió en escuelas de todo su país investigación de este tipo para examinar la práctica en su Plan de Mejora, a la vez, para estudiar los efectos de la evaluación en educación. Y esto no es todo, los EC han alcanzado un amplio proceso investigativo, en la medida que se observa realidades sociales de interés general, las cuales se despliegan desde una amplia gama de campos y enfoques que nutren la experiencia investigativa, a lo anterior se le añade, que los EC además de abordar un problema, recoger datos, plantear un marco teórico, formular hipótesis y analizar, han sido referente para estudios que pretenden explicar cómo opera la economía. Precisamente por esto, los EC son una ruta para el entendimiento de la política comparada, ya que la comparación

1 EC: sigla con la que se hará alusión durante el desarrollo del escrito al estudio de Caso. 
como lo mencionan (cfr. Lucca y Pinillos, 2015, p.4), es una herramienta para la construcción del conocimiento por su valor descriptivo, explicativo e interpretativo de la realidad.

$\mathrm{Al}$ respecto, los métodos comparados, surgieron para mirar más allá del caso único y poder comparar muchos casos, por eso, su principal fundamento es el campo de la Ciencia Política donde comparar "tiene el doble propósito de apuntar escenarios de concreción que permitan elevar los niveles de eficacia gubernamental al tiempo que determinar la consistencia institucional" (Oliva, 2008, p. 83). De ahí, surgen estudios desde el método comparado que buscan el análisis de lo político en temas como la construcción nacional, doctrinas políticas, la participación pública en el diseño constitucional, orden político, control del poder y gobernación en la emergencia, entre tantos, pero también se concede gran atención a procesos sociales como las organizaciones sindicales y las movilizaciones sociales, por no mencionar más.

Los anteriores antecedentes se convierten en el pretexto del presente artículo de reflexión teórica que tiene por objetivo analizar los principales elementos que constituyen el método comparado, para ello, desarrolla una triada teórico-metodológica que puntualiza sobre: los elementos de la comparación y el método comparado, la metodología de la comparación y recomendaciones para investigaciones basadas en el método comparado. Finalmente se presentan las conclusiones y la bibliografía.

\section{Elementos de la comparación y el método comparativo}

Para desarrollar este apartado, cuatro preguntas giran en torno a la cuestión: ¿Qué es la comparación? ¿Qué es comparable? ¿Cómo comparar? Y ¿Qué es y qué caracteriza el método comparado? Iniciemos el recorrido con la pregunta ¿Qué es la comparación?

Trayendo a colación a la Real Academia española, comparar consiste en fijar la atención en dos o más objetos para descubrir sus relaciones o estimar sus diferencias o sus 
semejanzas. De este modo, puede decirse que la acción de comparar tiene carácter científico, ya que determinar divergencias y similitudes entre objetos implica desplegar operaciones mentales como observar, analizar e interpretar, elementos que permiten descubrir significados y construir conocimientos. En consonancia con lo anterior, (cfr. Piovani y Krawczyk, 2017, p.822) consideran la comparación como actividad cognoscitiva y rasgo central de la actividad científica, que nos permite producir conocimiento y establecer relaciones con los otros y con el mundo. Por otro lado, comparar como lo señala Sartori (1994) es “asimilar y diferenciar en los límites” (p.35). Lo primero para asemejar dos objetos entre sí, y lo segundo, para identificar lo que hace que un objeto sea diferente de otro, de ahí, surge otro concepto que nos dice que para comparar se debe fijar parámetros y emplear categorías de análisis que sean derivadas de una teoría general y que permitan asimilar y diferenciar los objetos. Otro punto de vista señala que:

(...) Comparar permite, además, alcanzar otros fines: a) por medio de la analogía, similitud o contraste, a partir de lo conocido, comprender lo hasta ahora desconocido (es la llamada comparación pedagógica); b) remite a nuevos descubrimientos o resalta lo especial (comparación heurística), y c) al acentuar precisamente la diferencia, ayuda a sistematizar (comparación sistemática), aun cuando lo característico del objeto de estudio no se toma como singularidad, sino como especificidad. Estos tres fines, que sirven especialmente a la ciencia política, son importantes en la lógica de la comparación como método científico. (Nohlem, 2020, citando a Grosser, p. 43)

A razón de lo anterior, comparar nos mueve al desarrollo de operaciones mentales que muchas veces de modo implícito o inconsciente en la actividad cotidiana lo hacemos ya que es un ejercicio básico, sin embargo, comparar como actividad científica, permite conocer el grado de generalización del tema de investigación, explicar la génesis que impulsa a investigar, así como "elaborar un diseño de investigación con el que podamos hacer comparaciones y extraer de ellas las lecciones o bien las "recetas" que mejor se adapten” (...) para alcanzar los objetivos de estudio e investigación que nos planteamos” (Morlino, 2010, p.p. 11-12). 
Otra postura es la de Landman (2011), quien señala que comparar "es una actividad humana natural. Desde la Antigüedad hasta nuestros días, los individuos han tratado de comprender y explicar las similitudes y diferencias que perciben entre sí mismos y los demás” (p.27). Precisamente por esto, el ser humano realiza comparaciones sencillas para reconocer semejanzas y diferencias entre las mismas personas, de ahí, en la familia se compara a los hijos para determinar cual es igual al papá o a la mamá enfocados en los rasgos físicos o comportamentales, al mismo tiempo, se confrontan modelos educativos para determinar cual ejerce mayor influencia en el desarrollo de los estudiantes, a la vez, se comparan las actitudes de las personas para determinar como inciden en el comportamiento humano, y en un nivel más macro, se compara el manejo dado a la pandemia desde las entidades gubernamentales que pueden ser, entre municipios, departamentos y naciones para determinar los efectos en el desarrollo micro y macro, aspecto que reafirma el planteamiento de Landman (2011) “en definitiva, comparar es humano” (p.27).

Así mismo, la comparación es una "estrategia analítica con fines no solamente descriptivos sino también explicativos, un procedimiento orientado por sobre todo a poner hipótesis a prueba" (Pérez, 2007, p.1). Con esto, se quiere decir que la comparación como actividad cognoscitiva permite desde el análisis detectar en las variables de comparación aspectos como: sus semejanzas y diferencias, sus fortalezas y debilidades, y las oportunidades para la trasformación. Y si se trata de poner a prueba las hipótesis, su importancia radica en lo siguiente:

Favorecen la descripción y explicación: mediante las hipótesis el investigador anticipa cuáles son los elementos constitutivos del fenómeno bajo estudio, lo que contribuye a describir sus atributos o variables a partir de los valores y cualidades que los mismos poseen. También las hipótesis pueden establecer cómo se relacionan esos atributos, tarea que favorece la explicación. Cada vez que una hipótesis en estado de prueba recibe evidencia empírica en su favor o en su contra, nos informa algo acerca del fenómeno. Si la evidencia es a su favor la información sobre los fenómenos se incrementa. Aún si la evidencia es en contra descubrimos algo acerca del fenómeno que no sabíamos antes, con lo cual se expande el conocimiento del objeto. (Espinosa, 2018, p. 127) 
Ahondando un poco más, comparar tiene su razón de ser: algunos comparar para clasificar casos y objetos pertenecientes a una misma clase, otros lo hacen para desarrollar operaciones mentales que permitan deducir las semejanzas y diferencias de los objetos, y de los casos, también, hay quienes comparan para generalizar y particularizar sobre los objetos o casos, al respecto, comparar como método de investigación tiene objetivos concretos que radican en realizar descripción contextual, construir clasificaciones y tipologías, verificar hipótesis, y, predecir.

Comparar para realizar descripción contextual tiene como premisa describir los fenómenos políticos de un país o de países y se realiza de manera detallada con el fin de adquirir más conocimiento de la realidad contextual y profundizar para dar explicaciones de niveles superior (Masagualli y Bolaño, 2017; Salvatori y Terrón, 2019). Por otra parte, comparar para clasificar consiste en organizar por categorías para facilitar el hallazgo de las diferencias y semejanzas en las unidades de análisis, mientras que verificar hipótesis permite estudiar los factores que explican lo descrito y lo clasificado, como puede verse, son procesos continuos que dependen unos de otros para realizar la comparación, y, en cuanto a comparar para predecir conlleva a formular lo que puede ocurrir a futuro mediante generalizaciones.

Dicho esto, entonces: ¿Qué es comparable? Al respecto, hay que decir que la comparación busca identificar aquello que es bastante similar, o, por el contrario, distinto. En otras palabras:

Si dos entidades son iguales en todo, en todas sus características, es como si fuesen la misma entidad, y todo termina ahí. A la inversa, si dos entidades son diferentes en todo, entonces es inútil compararlas, y del mismo modo, todo concluye aquí. Las comparaciones que sensatamente nos interesan se llevan a cabo es entre entidades que poseen atributos en parte compartidos (similares) y en parte no compartidos (y declarados no comparables). (Sartori, 1994, p. 35) 
Hay que mencionar además que lo comparable es aquello que tiene algo en qué compararse, evitando de este modo errores como los señala la teoría Sartoriana que consisten en fabricar términos por: el desconocimiento de teorías generales, la mala clasificación de terminología, el abuso de la máxima según la cual todas las diferencias son diferencia de grado las cuales se extienden sobre un continuum de más-menos, y, el alargamiento de conceptos hasta el punto de convertirse en ideología.

Por lo tanto, lo comparable son unidades o casos confrontables, estos se refieren a "aquellos países que son objeto de análisis comparado" (Landman, 2011, p.43). En este sentido, podemos traer a colación a Skocpol (1984) sobre los estados y las revoluciones sociales desde una comparación histórica, donde "las revoluciones sociales son comparadas con los casos fallidos y con las transformaciones no social-revolucionarias" (p.23). El concepto de Skocpol sobre la revolución social, está vinculado con el cambio triunfal, convirtiéndose este en rasgo definitorio. Por ejemplo:

La Francia revolucionaria se convirtió de pronto en un poder conquistador en la Europa continental, y la Revolución rusa generó una superpotencia industrial y militar. La Revolución mexicana dio a su patria la fuerza política necesaria para convertirse en una de las naciones más industrializadas entre las naciones poscoloniales, y en el país de América Latina menos expuesto a asonadas militares. Desde la segunda Guerra Mundial, la culminación de un proceso revolucionario que llevaba largo tiempo en gestación ha reunido y transformado a la antes quebrantada China. Y nuevas revoluciones sociales han capacitado a países déscolonizados y neocoloniales, como Vietnam y Cuba, a romper las cadenas de una extremada dependencia. (Skocpol, 1984, p.19)

La comparación histórica de revoluciones, concretamente de Francia, Rusia y China, tuvieron su génesis en la política, porque los antiguos regímenes carecieron de alcance frente los desafíos de la época, de ahí, la autora hace un paralelo sobre la crisis revolucionaria de Francia y China, por no mencionar más, detallando que aunque ambos estados tenían una configuración muy diferente en sus estructuras sociales, 
las pautas estructurales y los procesos casuales fueron similares, lo que permitió sus caídas (Iñigo, 2019). Al mismo tiempo, los gobiernos francés y chino quedaron coaccionados por naciones extranjeras que lideraban en su momento lo económico, siendo esta otra similitud, y, la proliferación de grandes terratenientes en ambas naciones se convirtió en obstáculo para la organización que pretendían los estados.

Sin embargo, para la comprensión el ejercicio de comparación, en el estudio de las revoluciones no solo bastó con hacer análisis del estado, aquí se precisó, sobre la economía agraria, la organización del campesinado y el motivo de sus movilizaciones, punto de referencia para la comparación, destacándose de este modo que las movilizaciones de los franceses tuvieron por objetivo "las tradicionales organizaciones comunitarias de aldeas. En China, los campesinos participaron primero como bandidos sociales (...) en pos de objetivos concretos e inmediatos, no muy distintos de los que habían tratado de lograr en motines y rebeliones históricas “(Skocpol, 1984, p.188). Estas características del campesinado se convirtieron en variante explicativa de la comparación, pese a esto, lo que verdaderamente daba sentido a la comparación era la cuestión sobre qué transformaba al campesinado.

En la actualidad lo comparable a nivel investigativo, ha venido superando la frontera de Estado-Nación como unidad de análisis, ya que encontramos desde diversos ámbitos estudios comparados, de los más reciente, desde las Relaciones Internacionales Taborda y Riccardi (2019), estudian los casos de la cooperación para la paz de Colombia en una temporalidad que va del año 1998 al 2016, donde los principales aliados fueron los Estados Unidos y de la Unión Europea, dejando ver desde un análisis estadístico y documental la diferenciación de los dos socios en políticas de cooperación por razones como: Estados Unidos privilegiaba el recurso para refuerzo militar mientras que la Unión Europea su ayuda estaba destinada para la población civil; los Estados Unidos cooperaban como parte de su política exterior enfocada en seguridad nacional y la defensa del hemisferio occidental, por no decir más; mientras que la Unión Europea 
su cooperación tenía como fin consolidar la paz positiva que pone su mirada en la democracia incluyente y el desarrollo humano ( cfr. Taborda y Riccardi, 2019, p.123).

Otros casos que nos permiten reconocer estudios comparados de acuerdo con Morlino (2014), encontramos: "capacidad de gobierno y reformas institucionales en Italia; democratización en Europa Oriental, la fuerza del Partido Comunista respecto al Socialista en Italia tras la Segunda Guerra Mundial hasta 1991; responsabilidad de los gobernantes y capacidad de castigo de los gobernados” (p.65). Estos ejemplos sirven como medio para señalar que lo comparable formula conceptos empíricos y da paso a la formulación de hipótesis, de este modo, es posible comparar casos diferentes siempre y cuando se ubiquen dentro de una misma categoría.

Habiendo hablado de lo que es comparable, como lo son los casos, ahora nos adentramos en otras tres dimensiones de la comparación, la primera dimensión se refiere las unidades de análisis, definidas como "los objetos sobre los que se recaban datos, como por ejemplo individuos concretos, países, sistemas electorales, movimientos sociales, etc.” (Landman, 2011, p.43). De las unidades de análisis hay que decir que pueden ser únicas o múltiples, nos permiten realizar inferencias de nivel validas.

La segunda dimensión está ligada a las propiedades o variables que se van a analizar y se constituyen en el "conjunto de características o "aspectos» de las unidades o casos que se consideran relevantes para la investigación. Desde un punto de vista lógico-formal, las informaciones relativas al objeto de una investigación” (Morlino, 2014, p.57). Esta información respecto al objeto de investigación es importante para la formación de conceptos empíricos puesto que no solo facilita la comprensión del fenómeno desde sus atributos, sino que permite "identificar las variaciones del fenómeno en las distintas realidades. La «lección» de Sartori sobre este punto es inequívoca: el "perro-gato» existe principalmente por defecto de conceptualización (el sobreestiramiento de los conceptos) y de clasificación (precisamente la mala clasificación). (ob. Cit). 
De las variables también se dice que "son aquellos conceptos cuyos valores cambian en el seno de un conjunto de unidades determinado, tales como la renta, la identificación partidista, la tendencia a participar en movimientos de protesta, etc.” (Landman, 2011, p.43). Las variables pueden ser numerosas se definen mediante datos cualitativos o cuantitativos dependiendo su sentido estricto o clasificación. La tercera dimensión hace alusión a las observaciones que son el valor que poseen las variables en cada unidad de análisis, estos atributos pueden ser numéricos, verbales o visuales. Para puntualizar sobre estas dimensiones vale la pena reforzar mediante el siguiente ejemplo:

(...) Un estudio hipotético de los movimientos sociales en el Reino Unido, Francia, Holanda y Alemania podría tener una variable denominada, "estrategia", que incluyera las categorías "actuación a través de grupos de presión”, "manifestación pacífica”, "acción violenta directa”, "organización de base” y "labor de concienciación”. En tal estudio hipotético, los países serían los casos, las unidades de análisis los movimientos sociales, la variable sería la "estrategia", y la observación, el valor de la variable "estrategia" para un movimiento concreto en un país determinado. (Landman, 2011, p.43)

Ahora bien, ¿Cómo comparar? Para ello, es necesario adoptar una de las siguientes estrategias comparativas: "encontrar entidades similares en todas las variables excepto en una, (...) en aquella variable que nos interesa investigar. Por el contrario, (...) sistemas que difieren en la medida de lo posible, en todo, salvo en el fenómeno que se investiga” (Sartori, 1994, p.42). En consecuencia, se compara para explicar e interpretar, para perfilar nuevos conocimientos, para destacar las peculiaridades de los fenómenos conocidos, y, sistematizar la información distinguiendo las diferencias con fenómenos o casos similares, de lo contrario, no hay comparación.

Para hacer mas hincapié en como comparar, hay que tener en cuenta los siguientes mecanismos analíticos mencionados por Morlino (2014): -. El triángulo de Ogden y Richards. -. Las reglas de la conceptualización. -. El árbol de Porfirio. -. Estrategias clasificatorias, entre otras. 
Elmecanismo analítico del triángulo de Ogdeny Richards fue planteado en 1946, su propuesta radica en determinar los componentes de un concepto para establecer relaciones que incluyen una triada así: "el término (el vocablo utilizado), el significado vinculado y el referente empírico (el objeto al que remite la palabra) con un triángulo" (Morlino, 2014, p. 82). Esta consideración está muy asociada a la premisa Sartoriana de evitar estiramiento de conceptos por desconocimiento de teorías, esto, porque Ogden y Richards proponen el triangulo para evitar problemas de ambigüedad, vaguedad, banalidad, el desorden de conceptos en términos de una adecuada comparación, la tensión entre referente y significado, términos con el mismo significado o diferente significado. La razón de los anterior, porque a nivel científico se procura que cada termino sea un concepto con definición propia.

El mecanismo analítico de las reglas de la conceptualización sigue con la línea Sartoriana que aconseja: buscar las raíces de la terminología para que se respete su significado original y no se olvide su referente histórico, es decir, téngase en cuenta el uso que se ha dado a la palabra y su evolución en el tiempo, finalmente, advierte que al tener en cuenta los precedentes mencionados, no hay que olvidar que los conceptos deben estar vinculados a un campo semántico.

En cuanto al mecanismo analítico del árbol de Porfirio, la consideración es tener en cuenta la connotación y denotación del concepto, donde la primera hace mención al "conjunto de características definitorias esenciales y propiedades que caracterizan dicho concepto" (Morlino, 2014, p.89). Mientras que la segunda, remite a los referentes empíricos a los que aplica el concepto. De este árbol de abstracción también puede decirse que desarrolla un proceso que va de lo general a lo particular y viceversa, dejando ver los vínculos entre connotación y denotación, aquí es importante señalar que si la clasificación de términos y conceptos no es pertinente por pretender connotar y denotar al tiempo puede llegarse al estiramiento conceptual como lo señala Sartori.

El árbol de Porfirio desarrolla una escala de abstracción que facilita fines cognitivos y recognitivos y aporta a la clasificación que va de lo genérico a la diferencia. 
Asimismo, tiene otro fin de carácter explicativo que es la parametrización "operación necesaria y esencial con la que se hace constante un factor para analizar la variación de otros factores. La parametrización se utiliza en estadística, pero sobre todo en política comparada” (ob. Cit).

El mecanismo analítico de las Estrategias clasificatorias busca tener en cuenta unas reglas para el desarrollo de la operación mental, con el fin de que al hacer extensible un concepto se tengan en cuenta unas pautas así como un numero de clases para el mismo. De ahí, la primera regla es tener en cuenta la relevancia del criterio, aquí se busca definir las dimensiones primordiales de lo que se estudia, al respecto, se dice que un criterio resulta discriminador y significativo, si los casos estimados se distribuyen con bastante regularidad entre las clases resultantes. Otras reglas para tener en cuenta de acuerdo con Mill (1843) son:

(...) La exclusividad y la exhaustividad. La primera (exclusividad) supone que la clasificación debe formularse de tal forma que una determinada realidad debe pertenecer exclusivamente a una clase y no puede pertenecer al mismo tiempo a otra. Dicho de otra forma, el criterio distintivo que se asume como esencial en una determinada clasificación debe tener un fuerte poder discriminador. La segunda regla (exhaustividad) supone que cada clase que surja de la articulación de dicho criterio debe comprender todos los objetos o realidades asumibles. (Mill citado por Morlino, 2014, p.96)

Este mecanismo analítico de las Estrategias clasificatorias tiene mucho para ahondar, por ejemplo, sobre el nivel de abstracción de las clasificaciones y las tipologías, así como de la simplificación que surge de las mismas, ya que pueden reducir realidades complejas trayendo como consecuencias hacer perder información empírica valiosa.

Avanzando en el asunto ¿Qué es y qué caracteriza el método comparado? Iniciemos diciendo que es una forma de realizar de modo sistemático, organizado y estructurado un estudio investigativo, y según Lijphart (1971), "es un método de descubrimiento de relaciones empíricas entre variables” (p.218). Es decir, el investigador al entrar en contacto directo con la realidad 
y basado en la práctica y la observación de los objetos, construye conocimiento, entonces, una de sus características es que el investigador es un "pensador consciente como "el hombre que se da cuenta de las limitaciones de no tener un termómetro, sin embargo, puede decir mucho simplemente con caliente y frío, más cálido y templado"' (ob. Cit). También, es un procedimiento que permite realizar comparaciones explicitas y conscientes, entonces se caracteriza por "Dar respuestas a problemas del conocimientos natural y social” (Piovani y Krawczyk, 2017, p.822). Para ilustrar mejor, es normal que en la vida cotidiana comparemos para dar respuesta a problemas de conocimiento natural y social, con ello, buscamos establecer las diferencias y semejanzas de los objetos, donde la función de la comparación natural es confrontar operaciones simples, mientras que la comparación social se ocupa por contrastar operaciones complejas. Por ejemplo: un estudiante que ingresa a la carrera de Psicología trae consigo conocimientos que ha adquirido por experiencia directa, de ahí, está en capacidad de describir su propio comportamiento como el de los demás, a la vez, puede predecir las conductas de los demás mediante el desarrollo de operaciones simples que no han requerido de un adiestramiento formal.

Mientras que el mismo estudiante para adquirir un conocimiento social basado en problemas de comportamiento, ha de adiestrase en las dificultades de comportamiento más frecuentes, sus causas, las estrategias de intervención que faciliten mejorar el comportamiento, entre otras, de ahí, el desarrollo de operaciones complejas mediante el uso de instrumentos que le permitan conocer nuevas pautas de comportamiento para comparar conductas; entrevistar a sujetos que presentan comportamiento diverso, observar directamente a los sujetos para determinar los patrones de comportamiento más recurrentes y hallar semejanzas y diferencias, entre otras.

Continuando la línea: ¿Qué es y qué caracteriza el método comparado? Sartori (1994), lo plantea como un “"situar”, para aprender de las experiencias de los otros (...) para explicar mejor, y por otros motivos” (p.32). Al respecto, se caracteriza por orientarse a "personas concretas en contextos específicos (...) que pasa a tener consecuencias en la vida cotidiana de las personas que componen la sociedad” (De La Maza, De Cea y 
Rubilar, 2018, p. 15). De ahí, surge otro rasgo de los métodos comparados cuyo principal enfoque investigativo es el pensamiento político, semblanza que ha vislumbrado "procesos de modernización y de reforma del Estado impulsados desde mediados de los años 90 y que demandaron progresivamente la generación de conocimientos específicos en temas de políticas públicas y un proceso incremental de aprendizaje acerca de los mismos” (Maza et al., 2018, p.11). Por ejemplo, el Constitucionalismo en América Latina, para puntualizar como se crea una Constitución que funcione y perdure en el tiempo.

El método comparado al caracterizarse por abordar temas de orden político tiene su razón de ser en situaciones que se expresan en "“crisis": crisis de gobernabilidad, crisis de la política, crisis de las Ciencias Sociales. (...) pero paradójicamente la tragedia consiste en haber alcanzado un puesto académico, de importancia, profesionalizado y, dentro de lo posible conectado a la (...) investigación y teoría” (Pasquino, 1988, p, 34). Al respecto, cabe mencionar que su auge como lo detalla (cfr. Morlino, 2010 p.p.16-17), tiene incidencia desde clásicos como Descartes, Locke, Hegel, Comte, Durkheim, Weber, quienes catalogaron al método como la confrontación entre un "más-menos"; comparación con sentido espacial sincrónico; comparación con sentido temporal diacrónico y modo una manera de clasificar. También, el mismo autor evoca a contemporáneos como Almond, Sartori, Lijphart, que le han apostado a la comparación y la han explicado como: elemento central de la ciencia política, control de nuestras generalizaciones, y, relaciones empíricas planteadas como hipótesis entre variables lo cual tiene en cuenta: causas, efectos y aquellas variables de control.

\section{Metodología de la comparación}

El método comparado ha venido ejerciendo influencia en el campo de las Ciencias Sociales porque no se limitan a una estrategia particular y esto permite contribuir a la comprensión de los fenómenos estudiados. De ahí, el progreso en estudios como la educación comparada, el Constitucionalismo y teorías de desarrollo y democracia, a saber. A razón de lo anterior, conviene subrayar en este punto desde 
una perspectiva clásica, la propuesta investigativa de comparación denominada trans-contextual, la cual se despliega desde dos enfoques a saber: cross-national y cross-cultural, donde la primera (cross-national) según (cfr. Kohn citado por Piovani y Krawczyk, 2017, p.829), se caracteriza por utilizar metódicamente cuestiones o datos comparables de más de una nación, mientras que la cross-cultural, parte del interés por las culturas.

Por otro lado, ahondando un poco más desde el enfoque cross-national, (Lucca y Pinillos, 2015; Esteban, Amador, Mateos y Olmedo, 2019) la describen como un tipo de investigación comparada de dimensión horizontal relativa a lo espacial, que selecciona casos en el plano territorial, y, compara unidades nacionales (Fontalvo y Díez, 2021). Así mismo, las opciones metodológicas que proponen los autores para la perspectiva cross-national se fundamenta en cuatro opciones a saber: nacional, subnacional, supranacional y multinivel. Desde otra mirada a lo cross-national, se ha mencionado la prevalencia de cuatro tipos de investigación a saber: "a. Aquella en que la nación es el objeto de estudio; b. aquella en que la nación es el contexto de estudio; c. aquella en que la nación es la unidad de análisis; d. aquella que es transnacional” (Piovani y Krawczyk, 2017, citando a Kohn 1897, p. 830). Cada una desplegando sus propios intereses e iniciativas investigativas en el plano político y muchas de ellas desarrolladas por agencias internacionales.

También, Luca y Pinillos (2015), detallan la comparación desde una dimensión vertical, donde el espacio es la unidad de variación y el análisis está asociada al campo histórico para observar trayectorias y trasformaciones. Desde esta dimensión prevalecen tres modos de comparación: el primero denominado macro-causal, allí sobresale lo inductivo y la generalización, además busca analizar semejanzas y diferencias a partir de los casos. El segundo llamado demostración paralela de teoría, el cual combina historia y teoría, y, el foco de la comparación se da entre el caso y teoría, no, entre casos. El tercero relativo al contraste de contexto, donde se busca evidenciar la diferencia entre los casos por medio de estrategias conceptuales. 
Por otra parte, los mismos autores exhiben la comparación sincrónica cuyo propósito es dar cuenta de fenómenos recientes, ubicándose en la dimensión temporal con sentido cronológico, para evidenciar la simultaneidad del fenómeno en los casos por estudiar. Otra voz que se levanta para dar puntadas sobre la comparación sincrónica dice que:

El número de los casos es variable (de este modo también es eventualmente elevado) y las variables de contexto son difíciles de controlar, por lo que los esfuerzos se realizan por medio de una atinada selección en el contexto de casos parecidos (homogéneos), o mediante un enfoque de área. (Nohlem, 2020, p. 48)

Adentrándonos en la comparación diacrónica se puede decir que es la que versa sobre la discontinuidad de los tiempos sociales y políticos. Además "el número de los casos es reducido y las variables de contexto (aunque naturalmente están sujetas al cambio) son relativamente constantes" (ob-Cit).

De la comparación sincrónica y diacrónica Lucca y Pinillos (2015) expresan que pueden unirse para desarrollar una estrategia mixta de comparación, la cual se detiene en la semejanza de los casos y se caracteriza por la variabilidad en el número de casos, cabe aclarar que en estos tiempos este tipo de investigación el número de casos suele ser binario y “(...) las variables de contexto son en parte controladas, puesto que la selección de los casos se realiza muchas veces según la similitud en las variables de contexto”(Nohlam, 2020, p.48).

Para cerrar este apartado y retomando nuevamente a Lucca y Pinillos (2015) avanzamos en la comparación en Contextos de variables homogéneas y estudios de área, en esta perspectiva, la comparación tiene por criterio seleccionar una sola área geográfica, ya que se considera que en ella hay elementos semejantes desde alguna estructura social lo cual resulta determinante para encontrar fenómenos pertenecientes al nivel regional. Los autores se refieren también, a la comparación desde Contextos de variables heterogéneos 
o cross-área, aquí los casos pertenecen a distintas áreas geográficas y el interés por el estudio no está en los casos sino en el fenómeno que se pretende comparar.

\section{Recomendaciones frente a investigaciones desde la metodología comparada}

La vida cotidiana del ser humano está impregnada de comparaciones, por ejemplo: las amas de casa comparan el precio de los productos de la canasta familiar, los médicos compran la posología de los medicamentos para administrar a los pacientes aquella que contrarreste mejor la patología que presentan, los estudiantes comparan el estilo de enseñanza de los docentes, y así pasamos la vida de comparación en comparación y se va adquiriendo experiencia. Sin embargo, investigar desde una metodología comparada requiere que el investigador no tenga que recurrir al oráculo de Delfos para corroborar su hipótesis, de ahí, vale la pena recordar los estudios in vacuo o de un solo país, no desconocer y no desconocer que "las categorías de análisis pertenecientes a teorías generales" (Sartori, 1994, p. 37). Lo anterior, para evitar problemas de trasferencia ocasionada por fabricar términos y complicar las teorías que realmente nos interesan en el estudio, también, para impedir "el estiramiento o forzamiento conceptuales, esto es, hacia conceptualizaciones vagas y amorfas" (Sartori, 2008, p.22). En suma: mientras más se estiran los conceptos, menos capacidad tendremos de generalizar y verificar, o, por el contrario, falsear sobre una teoría.

Otra de las recomendaciones, es evitar "expediciones indiscriminadas para pescar datos" (ob. Cit). Con esto, se quiere dar a entender que el método comparado puede resultar afectado cuando se acumula información de manera indiscriminada, de ahí, los datos que se recojan ya sean desde lo cualitativo o cuantitativo, deben poseer suficiente poder discriminatorio, a la par, dice de lo cualitativo y lo cuantitativo que "ambas lógicas son complementarias y cada una tiene un campo legítimo de aplicación” (ob. Cit). 
Desde esta mirada puede pensarse en una triangulación de la información. ${ }^{2}$ Los anteriores razonamientos comulgan con los trazados de (Piovani y Krawczyk, 2017) quienes hacen los siguientes comentarios:

En términos metodológicos, es oportuno señalar que los estudios comparativos no se limitan a una estrategia en particular. En general son más frecuentes, o al menos tienen más difusión (...) las investigaciones centradas en el análisis secundario de datos estadísticos. Pero estas comparaciones estadísticas también se realizan a partir de datos primarios, en el marco de estudios que incluyen el diseño de instrumentos de relevamiento y de muestras, así como el trabajo de campo y la sistematización y análisis de los datos. No obstante, (...) no implica que no existan investigaciones comparativas que recurran a metodologías diferentes, incluso cualitativas, como las historias de vida o los estudios de caso. (Crossley y Vulliamy 1984, citados por Piovani y Krawczyk, 2017, p.828)

En cuanto a técnicas de investigación para las metodologías comparadas, se recomienda "las entrevistas y las observaciones en los contextos naturales - contenidas en la mayor parte de los estudios de caso en políticas públicas con algunas orientaciones más contemporáneas que incluyen trabajo con cartografías y aproximaciones prospectivas” (Maza et al., 2018, p. 14). De las entrevistas se sugiere que sean exploratorias o abiertas para informantes clave, a la vez, que se ajusten a las necesidades del equipo investigador, y precisamente aquí, las historias de vida juegan un papel importante ya que a veces es necesario pedir y a la vez permitir, que los entrevistados cuenten su historia o hablen de sus experiencias.

2 En la actualidad ha venido perdiendo fuerza la controversia epistemológica que nos ponía a debatir entre lo cualitativo y cuantitativo como método para resolver el problema de investigación, la razón de lo anterior, es porque ambos métodos facilitan las tareas cognitivas para dar respuesta a las necesidades humanas que son el punto de partida de toda investigación, de ahí, surge como posibilidad lo que hoy se ha venido configurado como la triangulación de la información, que tiene su alcance precisamente para resolver problemas de investigación mediante la combinación de ambos métodos. De hí, surgen recomendaciones para poder hacer esta combinación, entre ellas, que el investigador no se aferre a un solo método y tenga en cuenta la validez de cada uno, el abordaje del problema puede resultar mejor abordado, determinar un constructo puede haber convergencia de métodos, finalmente, ser moderados para evitar la saturación de la información. 
En cuanto a la observación, puede ser participante y no participante. Respecto a la Cartografía, puede decirse que enriquece la metodología comparativa ya que entre sus fines están: aproximarse a las comprensiones sobre el territorio que un conjunto de seres humanos posee de él; trabajo participativo y reflexivo sobre las diversas prácticas y problemáticas que aquejan un determinado contexto social e identificar las fortalezas y debilidades del territorio para plantear estrategias para transformar la realidad territorial, aspectos correlacionados con estudios de pensamiento político.

Otra técnica señalada por las autoras son los grupos de discusión desde el trabajo colaborativo e interdisciplinario, ya que en las metodologías comparadas resulta enriquecedor, investigadores e investigados con experiencia en diversas áreas de conocimiento puesto que las representaciones de la realidad resaltarían por su heterogeneidad. A lo anterior se le añade, que el ideal en estos grupos es que la discusión sea "generadora de significación y no solo de información, caracterizada de hecho por su no directividad" (ob. Cit).

Finalmente, parar responder a los objetivos de la investigación se sugiere el "uso flexible y combinado de distintos instrumentos y técnicas de recopilación de información" (ob. Cit). De ahí, los guiones de entrevistas hay que adaptarlos a los circunstancias y particularidades investigativas. Por otro lado, las observaciones tendrán que estar acompañados de diarios de campo que faciliten la sistematización de la información, y los grupos de discusión, con un taller que facilite la recolección de los significados que se pretenden para el estudio. Finalmente, para acompañar a la Cartografía, los talleres de mapeo colectivo deberán definir cuantos momentos de mapeo se requieren, así como las acciones concretas para lograr: ver: (que implica diagnosticar la realidad); juzgar: (que indica conceptualizar y profundizar sobre la realidad); y actuar: (que sugiere emprender acciones sociales trasformadoras para su realdad). 


\section{Conclusiones}

Proponer el método comparado como sustento investigativo antes que todo implica cuestionarse sobre: ¿Qué comparar? ¿Por qué comprar? Y ¿Cómo comparar? Al respecto hay que decir que comparar es una operación mental que todo ser humano desarrolla desde temprana edad y para diversos fines como observar, analizar, e interpretar. Pero si pretendemos desarrollar los anteriores procesos cognitivos, entonces, debemos determinar ¿qué comparar?, para decir que: comparamos semejanzas o atributos en parte compartidos con aspectos similares, y, diferencias de los objetos, en parte no compartidos, por ende, declarados no comparables, que nos permitan realizar generalizaciones para teorizar sobre la temática que se investiga, de ahí, el uso de parámetros y el empleo de categorías de análisis derivadas de la teoría general para evitar la fabricación de términos y alargamiento de conceptos por el desconocimiento de teorías generales.

A la par, el ¿cómo comparar?, debe conducir a explicar e interpretar, para perfilar nuevos conocimientos. Los anteriores precedentes sirven para decir que el método comparado es una forma de dar respuestas a problemas del conocimientos natural y social y que se caracteriza por aprender de las experiencias de los otros.

Por otro lado, es necesario definir la metodología de la comparación, pues existen varias vías que van desde la selección de casos pertenecientes a una misma área geográfica, en una temporalidad sincrónica y aquellos enfocados en abordar estudios conducentes en Estados-Nación. En este punto hay que señalar que, los estudios de metodología comparada requieren que revisemos las opciones que principalmente se han utilizado para construir los casos de análisis, como aquellos que permiten poner en evidencia cómo se han desarrollado otras opciones metodológicas, lo anterior, para aprender de las experiencias de los otros, como se mencionó en algún apartado de este escrito ya que pueden convertirse en nuevos prismas, sobre la política en la región. 
Finalmente, para el desarrollo de estudios desde la metodología comparada se recomienda la flexibilidad y la combinación de distintos instrumentos y técnicas de recopilación de información. A la vez, hay que adaptarlos a los circunstancias y particularidades investigativas, que faciliten la sistematización de la información y la recolección de los significados que se pretenden para el estudio.

\section{Referencias bibliográficas}

Agudelo Taborda, J., y Riccardi, D. (2019). La cooperación internacional para la paz en Colombia: los casos de Estados Unidos y de la Unión Europea (1998-2016). Geopolítica(s). Revista De Estudios Sobre Espacio Y Poder, 10(1), 107-134. https://doi.org/10.5209/GEOP.61477

Espinosa, E. (2018). La hipótesis en la investigación. Mendive. Revista de Educación, 16(1), 122-139. Recuperado en 15 de junio de 2021, de http://scielo.sld.cu/scielo.php?script=sci_arttext\&pid=S1815-76962018000100122\&ln$\mathrm{g}=$ es\&tlng=es.

Esteban, M., Amador, L., Mateos, F., y Olmedo, F. (2019). Mujer y medio ambiente. Una aproximación desde la acción socioeducativa. Collectivus, Revista de Ciencias Sociales, 6(1), 177-195. https://doi.org/10.15648/Coll.1.2019.10

Fontalvo, R., y Díez, A. (2021). Cartografías de la migración de retorno en Colombia. Evolución y patrones territoriales en el departamento del Atlántico. Collectivus, Revista de Ciencias Sociales, 8(1), 137-154. https://doi.org/10.15648/ Collectivus.vol8num1.2021.2959

Flyvbjerg, B. (2004). Cinco malentendidos acerca de la investigación mediante los estudios de caso. Revista española de investigaciones sociológicas, 106/4: 33-62. https://www. researchgate.net/publication/28148068_Cinco_malentendidos_acerca_de_la_investigacion_mediante_los_estudios_de_caso

Huertas, O. (2016). Emergentes conflictivos y desobediencia civil en las cárceles colombianas, miradas interdisciplinares. Advocatus, (26), 93-106. https://doi.org/10.18041/01240102/advocatus.26.936 
Iñigo, L. (2019). Educación para la preservación del medio ambiente. Collectivus, Revista de Ciencias Sociales, 6(1), 215234. https://doi.org/10.15648/Coll.1.2019.12

Landman, T. (2011). Política comparada. Una introducción a su objetivo y métodos de investigación. Madrid: Alianza editorial.

Lijphart, A. (2000). Modelos de democracia. Barcelona: Ariel.

Lucca, J., y Pinillos, C. (2015). Decisiones metodológicas en la comparación de fenómenos políticos Iberoamericanos. Disponible en: http://biblioteca.clacso.edu.ar/Espanaib/20161220051300/pdf_1455.pdf

Masagualli, N., y Bolaño, O. (2017). Alternativas de poder político en los movimientos sociales-rurales de Colombia: caso organización indígena del norte del Cauca. Collectivus, Revista de Ciencias Sociales, 4(2), 36-54. https://doi. org/10.15648/Coll.2.2017.3

Maza, F., Cea, M., y Rubilar, G.(2018). Estudio de caso en contextos interculturales e indígenas: perspectivas interdisciplinarias y comparativas del "Estado de acción" en Políticas indígenas y construcción del estado desde lo local: Estudio de casos del sur, centro y norte de Chile, págs. 11 - 35. CIIR, Centro de Estudios Interculturales e Indígenas: Pehuén. Santiago, Chile.

Morlino, L. (2010). Introducción a la investigación comparada. Madrid: Alianza Editorial.

Morlino, L. (2014). Introducción a la investigación comparada. Difusora Larousse - Alianza Editorial. https://elibro.net/es/ ereader/ucuauhtemoc/45438?

Nohlem, D. (2020). El método comparativo. En Sánchez, H. (ed.), Antologías para el estudio y la enseñanza de la ciencia política: La metodología de la ciencia política (pp 41-57).

Oliva, P. (2008). La ciencia política profesional. Revista Latinoamericana de Política Comparada, 1 (1), pp. 81-83.

Pasquino, G. (1988). Manual de ciencia política. Madrid: Alianza Editorial.

Pérez, A. (2007). El Método Comparativo: Fundamentos y Desarrollos Recientes. DOI: El Método Comparativo: Fundamentos y Desarrollos (studylib.es) 
Piovani, J., y Krawczyk, N. (2017). Los Estudios Comparativos: algunas notas históricas, epistemológicas y metodológicas. Educação \& Realidade, 42(3): 821-840: Disponible en https://www.scielo.br/pdf/edreal/v42n3/2175-6236edreal-42-03-00821.pdf

Salvatori, S., y Terrón, T. (2019). L’approccio intersezionale e quello transnazioanle nello studio dei flussi migratori: elementi per una proposta analitica. Collectivus, Revista de Ciencias Sociales, 6(1), 35-46. https://doi.org/10.15648/ Coll.1.2019.3

Sartori, G., y Morlino, L. (eds.). (1994). La comparación en las ciencias sociales. Madrid: Alianza.

Sartori, G. (2008). Falta de formación conceptual en política comparada. Revista Latinoamericana de Política comparada, 1 (1), pp.17-65.

Skocpol, T. (1984). Los estados y las revoluciones sociales. México: Fondo de cultura económico. 